\title{
MODELS FOR CALCULATING POSITION ANNIHILATION RATES IN SOLIDS
}

\section{O. OSIELE, I. A. FUWAPE and I. A. ASAOLU}

(Received 25 May 2001 ; Revision accepled 3 November, 2001)

\begin{abstract}
The local density approximation and the perturbed hypernetted chain approximation have been uscd to calculate the positron annihilation rate in some metals. Comparism of the theoretical techniques with experimental results shows that the two approximations agree with experiment for simple metals while the local density approximation agree better with experiments for transition metals. The perturbed hypernetted chain approximation is modified in this paper by considering the effects of electron-positron screening on the electron-positron amihilation rate. Results obtained from the modified model are in good agreement with experimental results for the alkaline, earth alkaline and transition elements.
\end{abstract}

Keywor'ss: Positron, annihilation rate, Screening and Melals.

\section{INTRODUCTION}

Over the years, positron annihilation techniques have been successfully used to study various properties of metals, alloys and semiconductors (Chabik et al, 1993. He el al, 1986 and Eldrup, 1995). Recently positron annihilation techniques have become a popular and useful tools for probing the structures of solids, liquid and alloys. It can be used to produce diffraction pattem of a defect (Mcmullen and Bishop, 1997 and Duke et al, 1977) and for the study of the density of electrons in momentum space of alloys that are in the icosahedral phase (Tanaka et al, 1997).

In using the positron annihilation teclunique, three experimental techniques are generally used. These are the positron lifetime technique, the angular correlation technique and the Doppler broadening technique. These experimental techniques measure properties of the gamma quanta released when a positron annihilates with an electron. The properties of these gamma quanta such as their energy, emission direction and time of emission which can all be measured provide useful information about the material in which the positron annihilates (Eldrup, 1995). In positron annihilation studies, it is possible to predict atomic and electronic structure of solids by careful analysis of the results obtained. Calculations give essential support for the analysis of the experimental result.

The independent particle model of approximation of Kahana (1963) does not give correct amnihilation rates as obtained by integrating the momentum density over the whole range of the momentum space. The discrepancy between the theoretical and experimental values was realized to originate from the strong positron - electron correlation that enhances the eflective electron density at the site of the positron. The Kahana formalisin shows the divergence of the annihilation rate at some relatively high value of the electron gas parameter, $r_{s}$, which is due to the fact that the Bethe - Goldstone equation does not treat properly the normalization of the two body wavefunction. In this approach, the annihilation rate excludes much of the polarization charge responsible for the effective interaction, which seems to make the resulting annihilation probability small in the metallic density (Kahana, 1963).

In the theory of Arponen and Pajanne (1979), the annihilation rate is a continuous function of the electron density at the positron site. This theory gave a good result espacially for the alkaline metals, but for metals of higher density, the theory does not agree well with experiments and the experimental values do not correspond to any particular pattern in terms of $r_{s}$. This is because in the theory of Arponen and Pajanne, a jellium was used without taking into consideration the inhomogeneity of the real lattice. Thus the strong internal electric field of a real lattice suppresses the elcctron-positron correlation, an effect which decreases the experimental annihilation rate in comparism with the jellium model. Furthermore, in real solids annihilation with core electrons tends to increase the annihilation rate when compared to the jellium model (Puska, 1991). The local density approximation takes into consideration the electron-positron correlation potential and the enhancement factor both to the first order approximation. These depend on the local density of the electron. Boronski and Niemenin (1986) gave the interpolation expression for calculating the enhancement parameter in the local density approximation. From which, the annihilation rate can be calculated. The Local density approximation over estimates the annihilation rate in the low-momentum range (Liming et al, 1997). Puska (1991) used the local density approximation to calculate the work function and annihilation rate in metals using self-consistent electron structure. He obtained a result that was in good agreement with experiment for alkaline and earth 
alkaline metals. Lowy and Jackson (1975) calculated positron annililation rate in solids using correlated electron wave function. The positron annihilation rate was in good agrement with the observed rates over the whole range of electron densities found in alkali metals using an effective interaction which includes the strong screening effects from one highly correlated screening, they calculated positron - elcelrun wave function. In the work of Lowy and Jackson, the divergence of the annihilation rate at relatively high values of $r_{s}$ is due to the neglect of the effective interaction between electron and positron. Partial annihilation rate of thermalized positron with electrons in a metal as a function of the initial electron momemtum was calculated by Lowy (1982). In the obtained result, only lithium of all the metals he investigated was in good agreement with experimental data. Furthermore, he calculated the electron - positron wave function self consistently using an effective interaction which includes strong interaction effects from one highly correlated screening electron at any instant of time.

Pietilainen and Kallio (1983) treated the position as a charged impurity in an electron gas. They obtained annihilation rates that are smaller than experimental ones for metals because the bound state is outside the metallic range.

A self consistent solution of the Kahana equation was obtained for a positron in an electron gas by Rubaszek and Stachowiak (1998). From the solution, they obtained the screening charge distribution around the positron. Using the screening charge distribution around the positron they calculated the positron annililation rate in metals. The annihilation rate was in reasonable agreement with experimental results at the high density limit (or low values of $r_{s}$ ). The divergence of annihilation rate a high values of $r_{\mathrm{s}}$ was as a result of the assumption on the state - independence of the trial function (Gondzik and Stachowiak, 1985).

In this work, electron-positron anniliilation rate in metals is calculated using the perturbed hypernetted chain approximation. The perturbed hypernetted chain approximations is a simple, efficient and direct method of calculating annihilation characteristics in metal lattices (Boronski and Stachowiak, 1998). Furthermore, the method allows lor a wider range of annihilation parameter calculations. Comparism of the result obtained with this model and the one obtained with the local density approximation with experiments is done. To improve the perturbed hypernetted chain approximation, an interpolation expression which puts into consideration electron-positron screening was introduced; The result obtained from the improved model is compared with experimental values.

\section{THEORY}

The rate at which positrons annihilate with surrounding electron is given by (Eldrup, 1985)

where $r_{0}$ is the classical radius of the atom, $n^{-}(r)$ and $n^{\prime}(r)$ are the total electron and positron density at $r, c$ is the speed of light in

$$
\lambda=\pi r_{0}^{2} c \int n^{-}(r) n^{+}(r) d r
$$

vacuum. The key observable signal is that the annihilation rate is proportional to the overlap of electron and positron densities. Where $n^{-}(r)$ and $n^{\dagger}(r)$ are known, the conventional way of calculating annihilation rates have been based on the application of the local-density formula (Boronski and Nieminen, 1986):

$$
\lambda=\pi r_{0}^{2} c \int n^{-}(r) n^{+}(r) g\left(r_{s}, 0\right) d r
$$

where $g\left(r_{s}, 0\right)$ is the enhancement parameter or correlation function. The enhancement factor takes into consideration the electron-positron interaction and is a manifestation of the electron-positron correlation. In the perturbed hypernatted chain approximation, the interpolation formula for the enhancement factor is given by (Stachowiak and Lach, 1993):

$$
g(r, 0)=1+1.23 r_{s}-0.1375 r_{s}^{2}+\frac{r_{s}^{3}}{6}
$$

Arponen and Pajanne (1979) in their formulation of the problem of a charged impurity gave the expression that can be used to calculate positron annihilation rate as:

$$
\lambda=\frac{12}{r_{s}^{3}} g\left(r_{s}, 0\right) \times 10^{9} \mathrm{~s}^{-1}
$$

and $r_{\mathrm{s}}$ for different elements is given as

$$
r_{s}=\left(\frac{3}{4 \pi n v}\right)^{3} \times \frac{1}{a_{0}}
$$


where $v$ is the valency of the element, $n$ is the electronic concentration and $a_{b}$ is Boln:"s radius. $g\left(r_{s,}, 0\right)$ takes different values in different model or approximation.

Since the positron is positively charged, it strongly distorts the electronic structure of the jellium (Arponen and Pajanne, 1979). The positron is an impurity which destroys the homogeneity of the electron gas (Pietilainen and Kallio, 1983). The presence of a positron in an electron gas will create an electric field round it which must be screened before it can annihilate with an electron. This will lead to the accumulation of screening change in the immediate neighbourhood of the positron and if small it contributes to the annihilation rate (Gondzik and Stachowiak, 1985). The way the positron is screened aflects its annihilation rate (Gondzik and Stachowiak, 1985). In the jellium model, $\lambda_{s}^{-1}$ is of the distance within which the potential is reduced about one third of its unscreened value, and is a measure of the effective range of the field due to the positron (Raimes.

$$
\varepsilon=e^{-\lambda}
$$

1963). In the presence of an impurity, the dielectric function of an electron gas can be expressed as (Inkson, 1984):

When screening is introduced into the perturbed hypernetted chain approximation to take into consideration the effect of screening between the electron and the positron before they annililate each other, we have the enhancement factor:

$$
g\left(r_{s}, 0\right)=1+1.23 r_{s}-0.1375 r_{s}^{2}+\frac{r_{s}^{3}}{6}+5.5 e^{-\lambda}
$$

where $\lambda_{\mathrm{s}}$ is the screening parameter defined as (Ashcroft and Mernin, 1976)

From equation (8), the screening parameter can be calculated which can be used to calculate the enhancement factor in equation

$$
\lambda_{*}=\frac{2.95}{r_{s}^{2}}
$$

(7) and hence annihilation rate using equation (4) above.

\section{RESULTS AND DISCUSSION}

Twenty - nine (29) elements consisting of alkaline, semiconductors and transition metals were used to test the models. The elements were chosen based on availability of experimental results for comparism with calculated values, industrial and technological applications of the metals and availability of the metals for experimental uses. Positron bulk annihilation rates in the 29 elements are shown in table 1.

As shown in table 1 and figure 1 , the local density approximation gave annihilation rates that close to but higher than experimental values of annihilation for metals of $r_{s} \geq 2.09$ apart from some cases of transition metals. But for metals for $r_{s} \leq$ 2.08 , the Local density approximation gave annihilation rates that are in a close agreement with experimental values (although lower). The perturbed hypernetted chain approximation (PIC $\wedge$ ) gave annihilation rates that were lower than experimental values for majority of the elements Investigated except for silicon, germanium, scandium and vanadium. The model is quite close to the experimental values for alkaline and earth - alkaline metals and the diflerence between experimental values and theoretical values for transition metals according to the perturbed hypernetted chain approximation is quite high. This is because the behaviour and properties of transition metals cannot be explained by the jellium model of solids wich is the basis of the perturbed hypernetted chain approximation.

The calculated values due to the local density approximation is closer to experimental values in all cases than the perturbed hypernetted chain approximation, this can be attributed to the fact that in the local density approximation calculations, the crystal structure of the metals are considered.

Although calculations due to the perturbed hypernetted chain approximation gave values that are in reasonable agreement with experimental values for alkali and earth-alkali metals; the approximation is simple, efficient and gives the hope for direct calculations of annihilation characteristics in metal lattices. Although calculations using the perturbed hypernetted takes a shorter time when compared to the local density approximation (Boronski and Stachowiak, 1998), Its agreement with experimental values for alkaline and earth-alkaline metals is because jellium models work well for these metals.

As shown in table 1 and figure 1 , the annihilation rates obtained with the improved perturbed hypernatted chain approximation (equation 7 above) is in perfect agreement with experimental values for metals whose $r_{s}$ lies between 3.25 and 5.60 and it approximates experimental values for other metals than the perturbed hypernetted chain approximation. 
Table 1: Positron annihilatien rate in some metals calculated using the perlurbed hypernetted clain approximation (PHC.A), Local density approximation (LDA), improved hypernetted chain approximation (IPHCA) and exper imental values (EXPT). The experimental values are taken from reference Danuik et al, (1991) excepi for si and Ge which were taken from Barbiellini et al, (1995)

\begin{tabular}{|c|c|c|c|c|c|c|}
\hline Element & $\begin{array}{l}\text { Crystal } \\
\text { structure }\end{array}$ & $r_{s}(a . u)$ & $\lambda_{\text {f:xp1 }}\left(10^{19} \mathrm{~s}^{-1}\right)$ & $\lambda_{\text {PHICA }}\left(10^{\circ} \mathrm{s}^{-1}\right)$ & $\lambda_{\text {f., }}\left(10^{19} s^{-1}\right)$ & 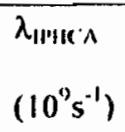 \\
\hline $\mathrm{Li}$ & $\mathrm{BCC}$ & 3.23 & $3 . \overline{44}$ & 3.24 & 3.28 & 3.62 \\
\hline $\mathrm{Na}$ & $\mathrm{BCC}$ & 3.93 & 2.96 & 2.73 & 2.97 & 2.98 \\
\hline $\mathrm{K}$ & $\mathrm{BCC}$ & 4.86 & 2.52 & 2.38 & 2.58 & 2.34 \\
\hline $\mathrm{Rb}$ & $\mathrm{BCC}$ & 5.20 & 2.46 & 2.31 & 2.53 & 2.44 \\
\hline $\mathrm{Be}$ & IICP & 1.88 & 7.04 & 7.10 & 7.30 & 8.25 \\
\hline $\mathrm{Mg}$ & $\mathrm{HCl}$ & 2.66 & 4.44 & 4.11 & 4.21 & 4.69 \\
\hline $\mathrm{Ca}$ & $\mathrm{FCC}$ & 3.27 & $\cdots$ & 3.22 & 3.26 & 3.57 \\
\hline $\mathrm{Al}$ & $\overline{F C C}$ & 2.00 & 6.13 & 5.98 & 6.02 & 6.94 \\
\hline$\overline{S i}$ & DIA & 2.00 & $4.6^{\circ}$ & 6.40 & 4.52 & 7.36 \\
\hline $\mathrm{Ge}$ & DIA & 2.09 & $\overline{4.36}$ & 5.91 & 4.39 & 6.58 \\
\hline Sc & $\| \mathrm{ICP}$ & 2.33 & 4.35 & 4.97 & 5.03 & $5 . \overline{73}$ \\
\hline $\mathrm{Ti}$ & HCP & 1.92 & 6.80 & 6.81 & 6.58 & 7.90 \\
\hline $\mathrm{V}$ & $\mathrm{BCC}$ & 1.64 & 7.69 & 9.13 & 6.02 & 10.57 \\
\hline $\mathrm{Mn}$ & CUB & 2.14 & $\cdots$ & 5.60 & $9.71)$ & 6.56 \\
\hline $\mathrm{Fe}$ & $\mathrm{BCC}$ & 1.84 & 9.43 & 7.32 & 9.90 & 8.52 \\
\hline $\mathrm{Co}$ & $\mathrm{HCP}$ & 2.08 & 8.45 & 5.95 & 10.30 & 6.91 \\
\hline $\mathrm{Cu}$ & $\mathrm{FCC}$ & 2.12 & 9.09 & 5.76 & 9.43 & 6.68 \\
\hline $\mathrm{Zn}$ & $B C C$ & 2.13 & 6.76 & 5.03 & 7.46 & 5.80 \\
\hline $\mathrm{Zr}$ & $\mathrm{HCP}$ & 2.11 & 6.06 & 5.81 & 6.30 & 6.73 \\
\hline $\mathrm{Nb}$ & $\mathrm{BCC}$ & 2.13 & 8.40 & 5.72 & 5.20 & 6.63 \\
\hline Mo & $\mathrm{BCC}$ & 1.84 & 9.71 & 7.36 & 9.01 & 8.56 \\
\hline $\mathbf{P d}$ & FCC & 2.28 & 10.42 & 6.66 & 9.71 & 5.91 \\
\hline$\overline{\mathrm{Ag}}$ & FCC & 2.40 & 7.63 & $\overline{5.13}$ & $8 . \overline{33}$ & 5.47 \\
\hline$\overline{C d}$ & $\mathrm{HCP}$ & 2.59 & 5.71 & 4.25 & 6.54 & 4.81 \\
\hline$P t$ & $\mathrm{ICC}$ & 2.01 & 10.10 & 6.51 & 10.64 & 7.33 \\
\hline $\mathrm{Au}$ & FCC & 2.39 & 8.55 & 4.77 & 9.35 & 5.49 \\
\hline
\end{tabular}




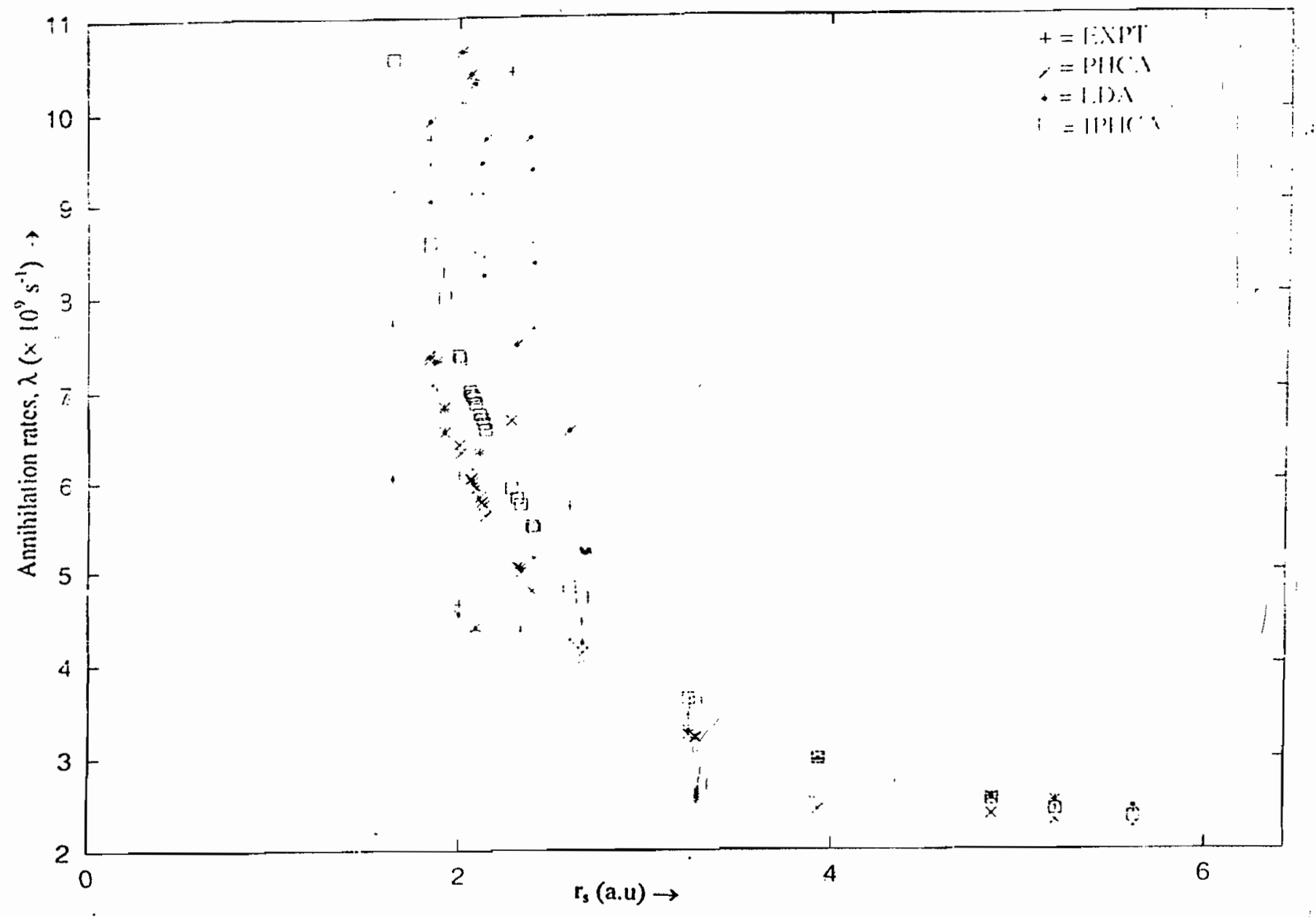

Fig 1: Variation of annihilation rate, $\lambda$ with electron gas paraneter, $r_{8}$ for different models. Where EXPT is experimental, PHC $A$ is perturbed hypernetted chain approximation, LDA is local density approximation and IPHCA is improved hypemetted chain approximation.

The inability of the above models to reproduce exactly experimental results can be attributed to some factors which experimental annihilation rates depends on an such as sample preparation, annealing of metals, timing device and data analysis procedure.

\section{CONCLUSION}

In this paper, we have calculated electron-positron annihilation rate in some selected metals using the local density approximation method and the perturbed hypernetted chain approximation. The two models gave results that were in agreement with experimental results while the local density approximation gave better result for transition metals. Since calculations with the perturbed hypernetted chain approximation is shorter and fast, an attempt is made to improve on the accuracy by introducing the idea of screening into the enhancement parameter. The improved perturbed hypernetted claain approximation gave a better result than the earlier ones showing that it is a better model than the other two and can be used as a model for predicting positron annihilation rate and can be used for probing atomic structure of solids. The model shows that introducing electron-positron screening gave a better annihilation rate results in metals that are in good agreement with experimental values.

\section{REFERENCES}

Arponen, J. and Pajanne, E., 1979. Electron liquid in collective Description iii: Positron anniluilation. Ammals of Iliysics. I21: 343 - 389.

Ashcrofi, N. W. and Mermin, N. D. 1976.Solid State physics. Holt, Rinehart and Winston, New York 342pp.

Barbiellini. B., Puska, M. J., Torsti, T. and Nieminen, R. M., 1995. Gradient correction for positron states in solids. Physical Review B.51(11):.7341 - 7344 . 
Bronski, E. and Stachowiak., H., 1998. Positron-electron correlation energy in an electron gas according to the perturbed -hypernetled chain approximation. Physical Review B. 57(11): $\quad 6215-6218$.

Clrabik. S., Rudzinskg, W., Szuszkiewicz, M. and Szymanski, C., 1993. Investigation of the solid-liquid phase transition for snln alloys by the positron annihilation method. ACTA physica polonica A. 83(3): $261-265$.

Daniuk,.S., Sob, M. and Rubaszek, A.., 1991. Theoretical calculations of positron annililation with rare-gas core electrons in simple and transition metals. Physical Review B. 34(4): 2580 - 2593.

Duke, C. B., Patron, A., Lazarides, A.., V asumathi, D. and Canter, K. F., 1977. Low-energy positron diffraction from CuTe(110):A minimun - variance R-Factor analysis. Physical Review B. 55(11): $7181-7189$.

Eldrup. M., 1995. Positron methods for the study of defects in bulk materials. Journal De Physique IV. 5: 93 -.- I09.

Gondzik . J. and Stachowiak, H., 1985. Screening of positive particles in Jcllium. Joumal of physics C: solid state physics 18: 5394 5413.

He, Y. S., Hasegawa. M., Lee, R. and Berko, S., 1986. Positron-annihilation study of voids in a-Si and a-Si: H. Physical Review B. 33(8): 5924 - 5927.

Inkson, J. C., 1984. Many body theory of solids. Plenum press, New York, 21 p.

Kahana, S., 1963. Positron annihilation in metals. Physical Review . 129(4): 1622 - 1628.

Liming, W., Panda, B. K., Fung, S. and Beling, C. D., 1997. Calculation of the momemtum distributions of positron annihilation radiation in Ge. Journal of Physics: Condensed matter 9: 8147 - 8154.

Lowy, D. N., 1982. Momentum - dependent annihilation rate for positrons in melals. Physical Review B. 20(1): 66 $-65$.

Lowy. D. W. and Jackson, A. D., 1975. Positron annihilation and electron correlations in metals. Physical ReviewB.12(5): 1689 1706

McMullen, T. and Bishop, M. F., 1997. Diffraction pattern of a defect: Two dimensional angular correlation of positron - annihilation radiation studies of defects in semiconductors. Physical Review B. 55( I I): 4046 -- 4049.

Pietilainen, P. and Kallio, A.., 1983. Hypernetted - chain theory of charged impurity with mixture formalism. Physical Review B. $27(1): 224-230$.

Puska, M. J., 1991. Ab-initio calculation of positron annihilation rates in solids. Journal of Physics: Condensed matter. $3: 3455-3469$.

Raimes, S., 1963. The wave mechanics of electrons in metals. Amsterdam, North Holland 195p.

Rubaszek, A. and Stachowiak, H., 1988. Self-consistent solution of the Kahana equation for a positron in an electron gas. Physical Review B. $36(6)$ : $3836-3853$.

Stachowiak, H., 1990. Electron-Positron interaction in jellium. Physical Review B. 41(18): $12522 \ldots 12535$.

Stachowiak, H. and Lach, J., 1993. Positron annihilation in an electron gas from low to high densities. Physical Review B. $48(13) .828$ -9830 .

Tanaka, Y., Nanao, S. and Tanigawa, S., 1997. Study of the density of electrons in momentum space in the AL-L.i lcosahedral phase by means of positron annililation. Joumal of Physics: Condensed inatter. 9: $11247-$ 11261 